\title{
SUPPORTING INFORMATION \\ for \\ Electric-Field Control of Neon Uptake and Release to and from Clathrate Hydrates
}

\author{
Yogeshwaran Krishnan ${ }^{\mathrm{a}}$, Mohammad Reza Ghaani ${ }^{\mathrm{a}^{*}}$ and Niall J. English ${ }^{\mathrm{a}^{*}}$ \\ ${ }^{a}$ School of Chemical and Bioprocess Engineering, University College Dublin, Belfield, \\ Dublin 4, Ireland
}

E-mail: niall.english@ucd.ie,mohammad.ghaani@ucd.ie

Table S1. Partial Charges and van der Waals Parameters

\begin{tabular}{|c|c|c|c|}
\hline Atom & $\boldsymbol{\sigma}, \boldsymbol{\AA}$ & $\boldsymbol{\varepsilon}, \mathbf{k J ~ m o l}^{-\mathbf{1}}$ & $\mathbf{q}_{\text {atom }}, \mathbf{e}$ \\
\hline $\mathrm{H}$ & 0.000 & $0.00 \times 10^{0}$ & 0.5564 \\
\hline $\mathrm{O}$ & 3.150 & $6.36 \times 10^{-1}$ & 0.000 \\
\hline $\mathrm{M}$ & 0.000 & $0.00 \times 10^{0}$ & -1.1128 \\
\hline $\mathrm{Ne}$ & 2.780 & $2.88 \times 10^{-1}$ & 0.000 \\
\hline
\end{tabular}

Arrhenius Fit

The relationship between apparent dissociation rate constant $k$ in Eq. 4 and temperature can be expressed by the Arrhenius equation (Eq. S1), where $A$ is the pre-exponential factor and $E_{a}$ $\left(\mathrm{J} \mathrm{mol}^{-1}\right)$ refers to the energy barrier of dissociation.

$$
k(T)=A \exp \left(\frac{-E_{a}}{R T}\right)
$$

Background on Thermodynamic Integration

The Helmholtz free energy, $A$, is related to the partition function $Q$ of an NVT ensemble, which is assumed to be the equilibrium ensemble generated by an MD simulation at constant volume and temperature. The generally more useful Gibbs free energy, $G$, is related to the partition function $\triangle$ of an NPT ensemble, which is assumed to be the equilibrium ensemble generated by an MD simulation at constant pressure and temperature:

$$
\begin{aligned}
& A(\lambda)=-k_{B} T \ln Q \\
& Q=c \iint \exp [-\beta H(p, q ; \lambda)] d p d q
\end{aligned}
$$


where $\beta=1 /\left(k_{B} T\right)$ and $c=\left(N ! h^{3 N}\right)^{-1}$. These integrals over phase space evaluated the derivative with respect to $\lambda$ as an ensemble average:

$$
\frac{d A}{d \lambda}=\frac{\iint(\partial H / \partial \lambda) \exp [-\beta H(p, q ; \lambda)] d p d q}{\iint \exp [-\beta H(p, q ; \lambda)] d p d q}=\left\langle\frac{\partial H}{\partial \lambda}\right\rangle_{N V T ; \lambda}
$$

The difference in free energy between A and B can be found by integrating the derivative over $\lambda$ :

$$
A^{B}(V, T)-A^{A}(V, T)=\int_{0}^{1}\left\langle\frac{\partial H}{\partial \lambda}\right\rangle_{N V T ; \lambda} d \lambda
$$

\section{$\underline{\text { Potential Energy vs. Time }}$}

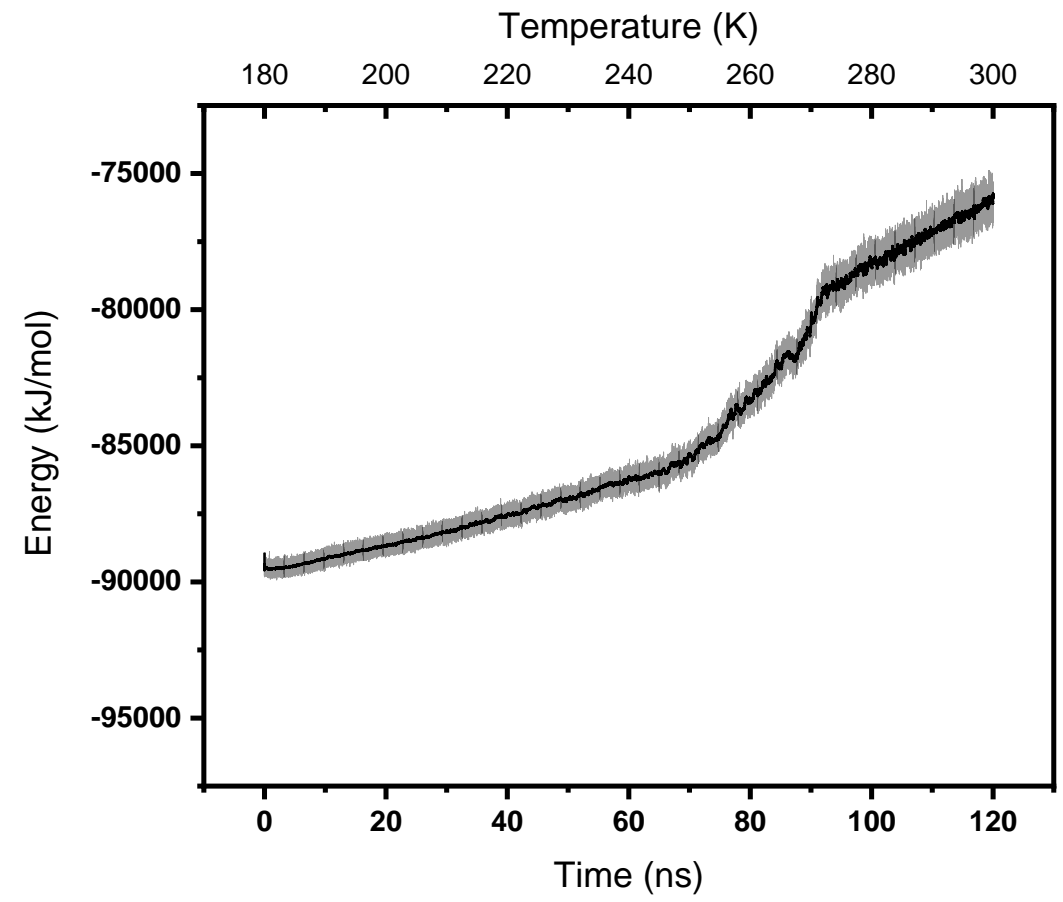

SI. 1 Potential energy with respect to temperature and time 
No. of hydrate-like water molecules and neon atoms

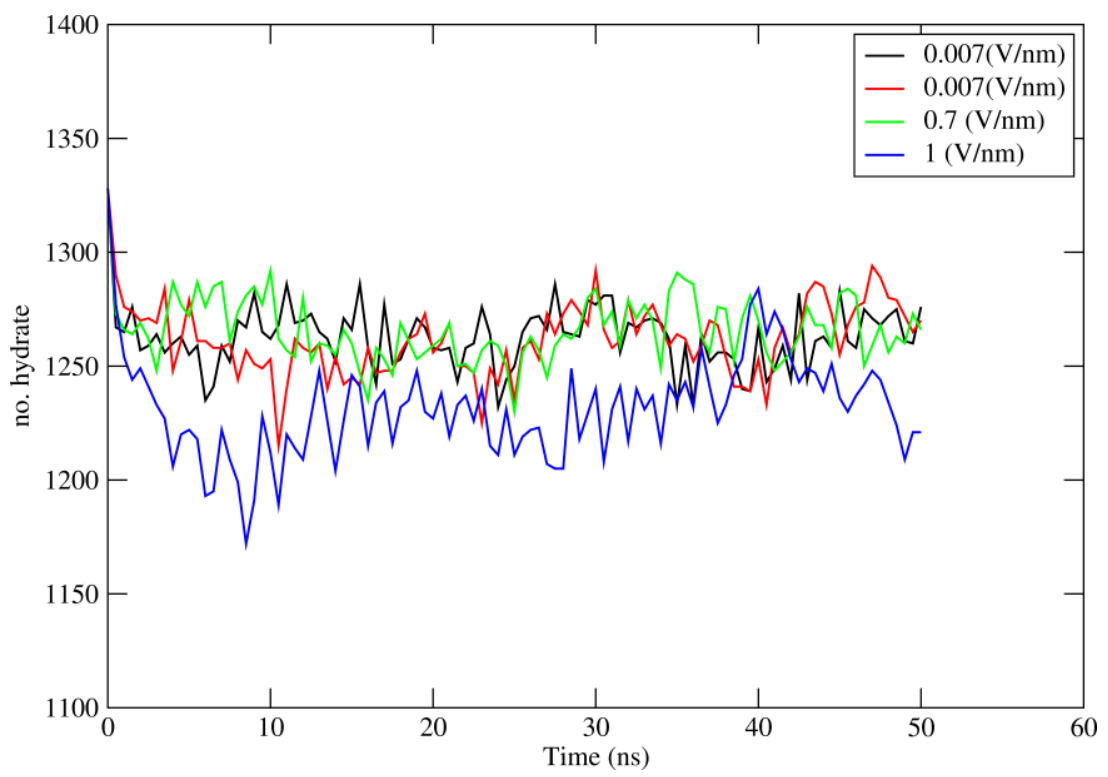

SI. 2 No. of hydrate-like water molecules in the applied electric field at $225 \mathrm{~K}$ (lattice preservation)

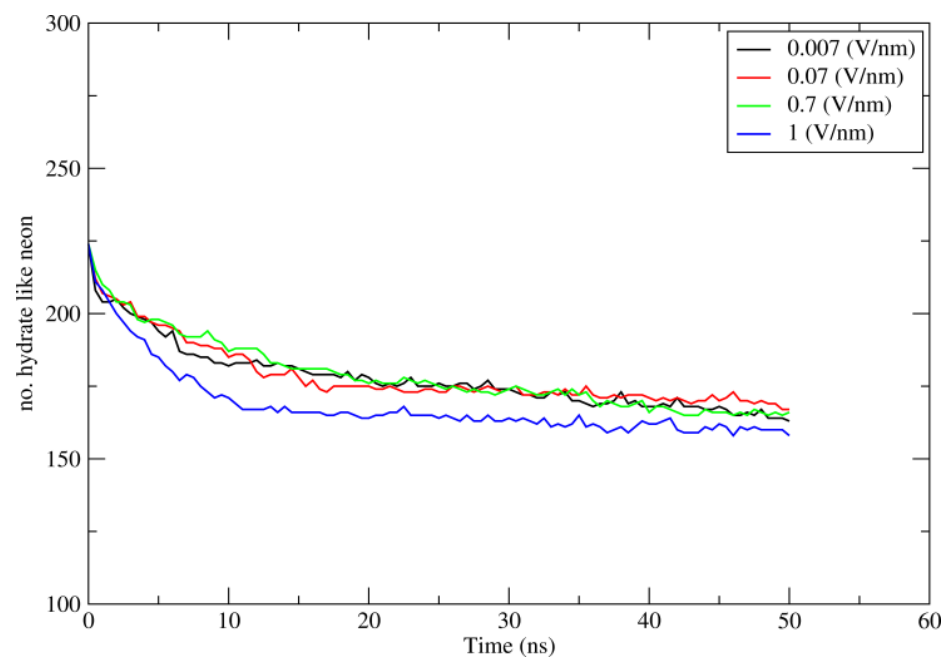

SI. 3 No. of hydrate-like neon atoms in the applied electric field at $225 \mathrm{~K}$ (leakage) 
$\left\langle\frac{\partial U(\lambda)}{\partial \lambda}\right\rangle$ for different values of $\lambda$

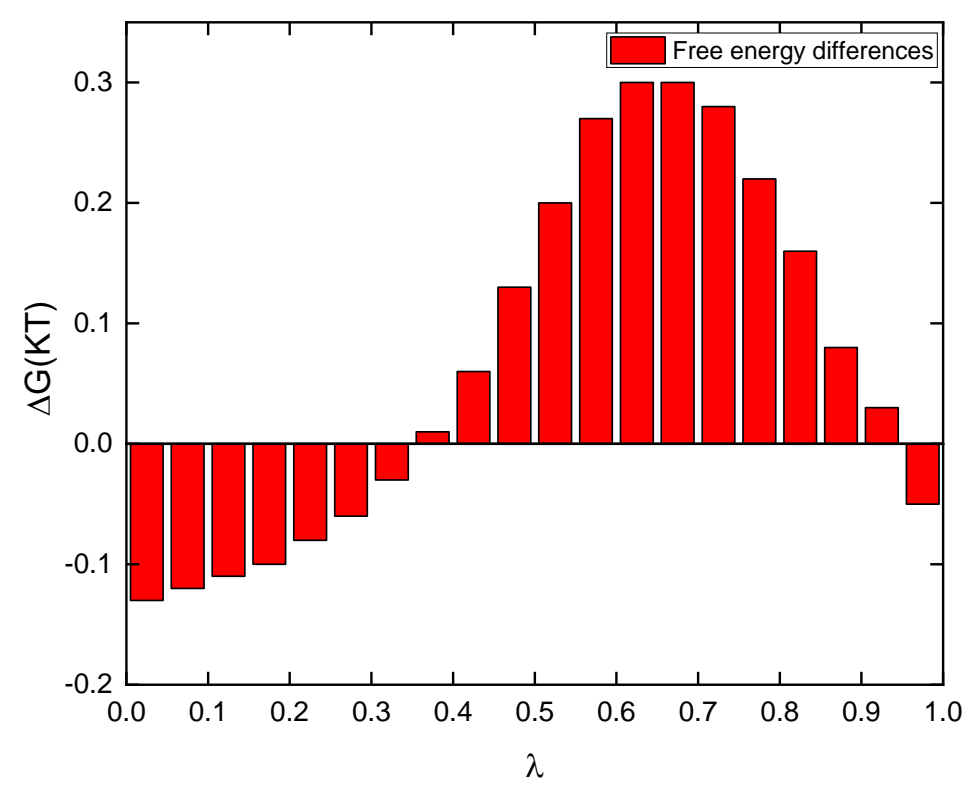

SI 4. A representation of $\left\langle\frac{\partial U(\lambda)}{\partial \lambda}\right\rangle$ for different values of $\lambda$ for neon substitution in the large cage of the clathrate hydrate. The integration of this curve gives $\Delta \mathrm{G}_{\mathrm{TI}}$ 


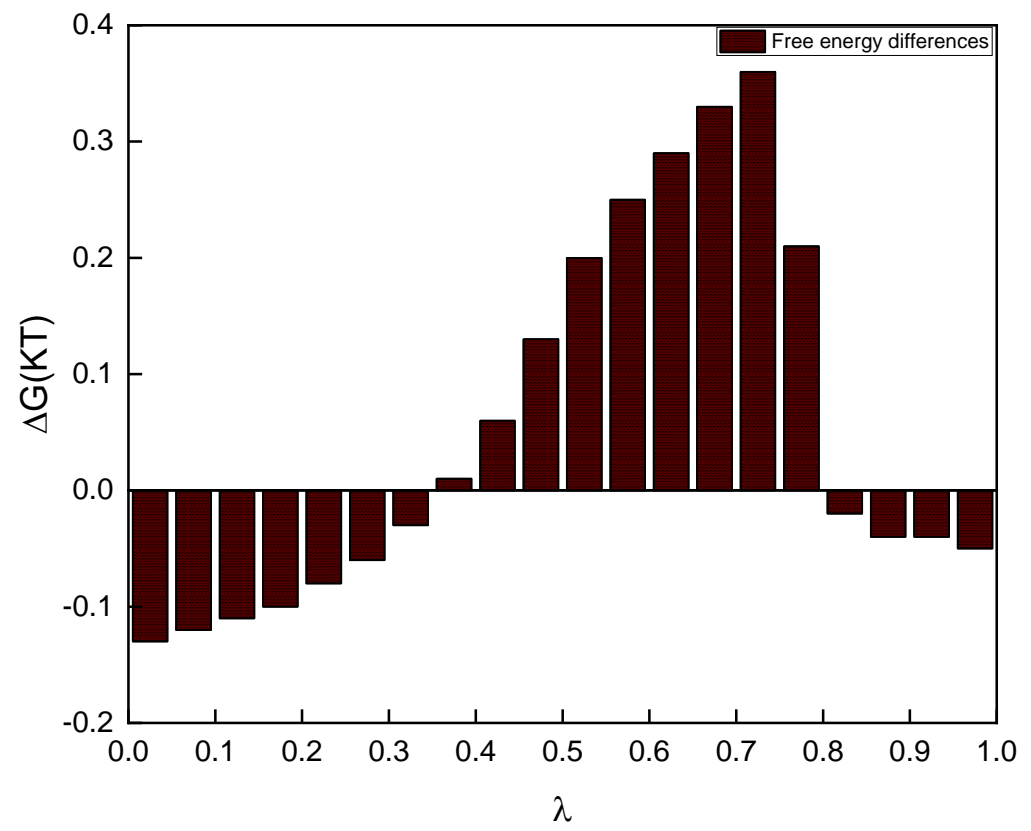

SI. 5. A representation of $\left\langle\frac{\partial U(\lambda)}{\partial \lambda}\right\rangle$ for different values of $\lambda$ for neon substitution in the small cage of the clathrate hydrate. The integration of this curve gives $\Delta \mathrm{G}_{\mathrm{TI}}$

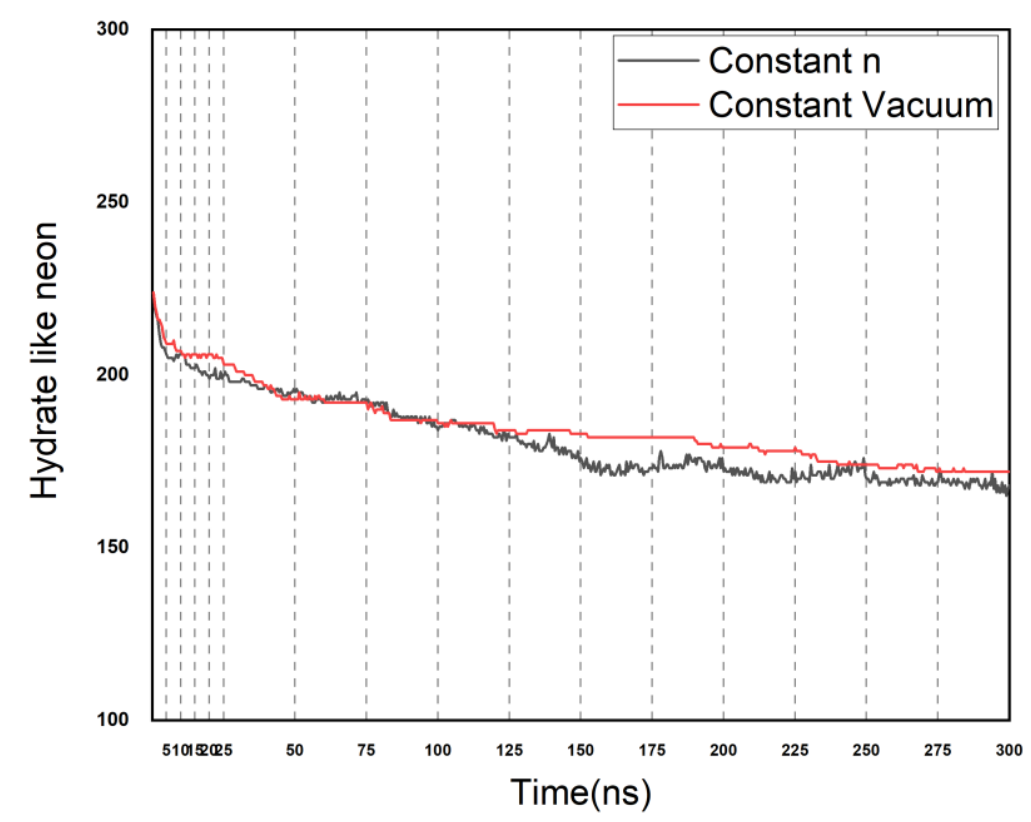

SI. 6. Neon-release kinetics for a constant number of moles and constant vacuum conditions, at $200 \mathrm{~K}$ (without an applied field) 


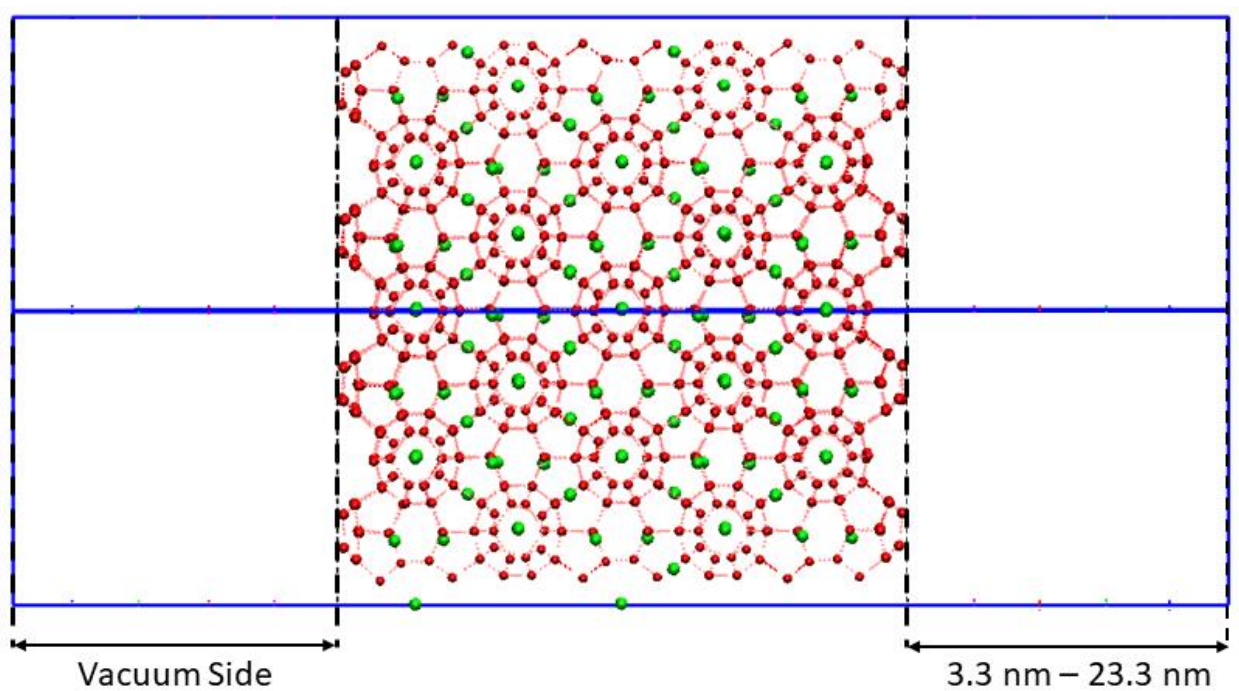

SI. 7. Designed simulation box, featuring different vacuum sizes

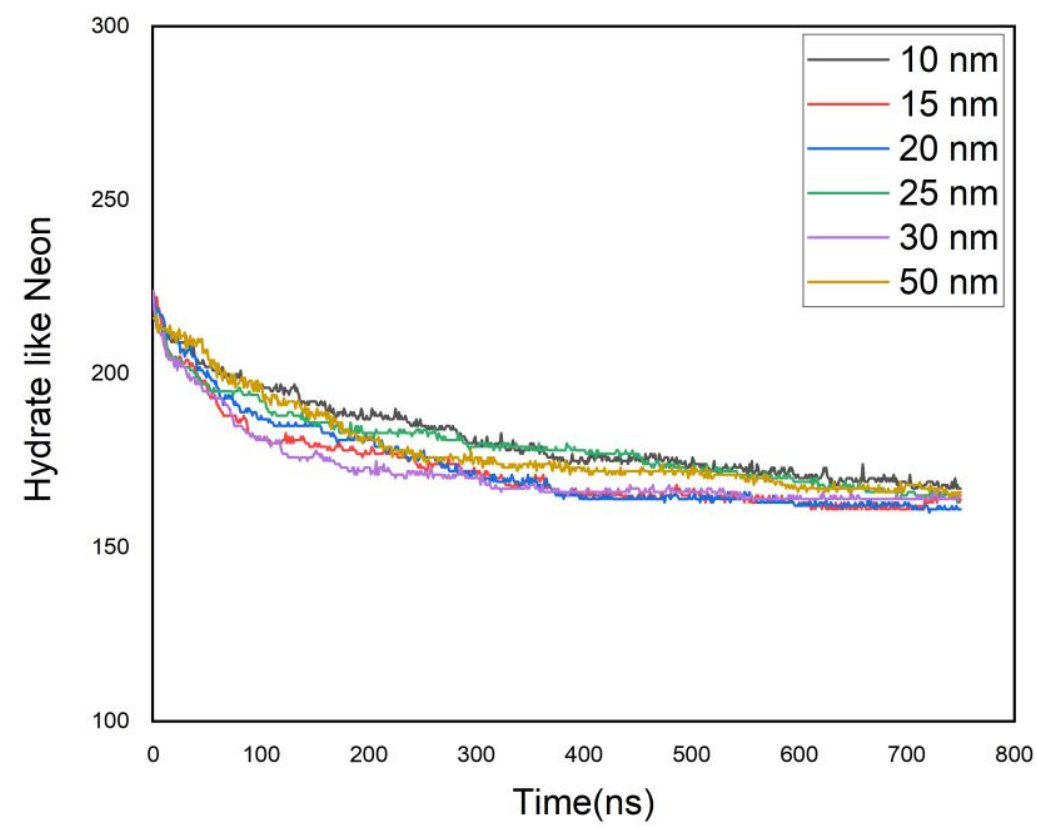

SI. 8. Neon-release kinetics at constant number of moles, but different vacuum size, at 200 $\mathrm{K}$ (without an applied field) 